\title{
A Preventative Respiratory Protocol to Identify Trauma Subjects at Risk for Respiratory Compromise on a General In-Patient Ward
}

\author{
Bethany A Nyland MD, Sarah K Spilman MA, Meghan E Halub MD, Keith D Lamb RRT-ACCS, \\ Julie A Jackson RRT-ACCS, Trevor W Oetting RRT, and Sheryl M Sahr MD MSc
}

\begin{abstract}
BACKGROUND: Patients are at risk for respiratory complications after sustaining blunt chest trauma, yet contradictory evidence exists about the utility of prophylactic respiratory therapy to reduce respiratory complications in this population. This study assessed the effectiveness of a proactive respiratory protocol on an in-patient ward to identify trauma patients at risk for pulmonary complications, administer appropriate therapies, and prevent deterioration requiring transfer to the ICU. METHODS: Trauma patients received a respiratory therapy evaluation at the time of admission to a general in-patient ward at a Level 1 trauma center. If subjects met protocol inclusion criteria, they received prophylactic respiratory treatments, primarily MetaNeb therapy, Vest therapy, or EzPAP. Multiple phases were included to evaluate the effectiveness of the protocol, with 50 subjects in each phase: a pre-protocol phase before adoption of the protocol; phase 1, which was found to have low physician adherence and overly broad inclusion criteria; and phase 2 , with improved adherence and narrower inclusion criteria. Study inclusion criteria mirror the protocol criteria from phase 2: $\geq 3$ rib fractures; pulmonary contusion; exacerbation of COPD, asthma, or other lung disease; or age $\geq 65 \mathrm{y}$ with expected immobility of $\geq 48 \mathrm{~h}$. RESULTS: The respiratory protocol was associated with an elimination of unplanned admissions to the ICU. After controlling for injury severity and other important clinical factors, receiving the protocol significantly decreased hospital stay by approximately $1.5 \mathrm{~d}$. More subjects were admitted from the emergency department directly to the ward, avoiding the ICU. Bronchodilator use also decreased, although the result did not reach statistical significance. CONCLUSIONS: Study results suggest that a preventive respiratory protocol had a beneficial effect on patient outcomes; receiving the protocol reduced hospital days and eliminated unplanned admission to the ICU. Key words: prophylactic respiratory therapy; metaneb; bronchodilators; trauma; chest injury; respiratory complications; stay. [Respir Care 2016;61(12):1580-1587. (c) 2016 Daedalus Enterprises]
\end{abstract}

\section{Introduction}

Trauma patients are at risk for respiratory complications after injury, including pneumonia, acute lung injury, ARDS, and unplanned intubation. ${ }^{1-10}$ Respiratory complications

Drs Nyland and Halub are affiliated with the General Surgery Residency Program, Iowa Methodist Medical Center, Des Moines, Iowa. Ms Spilman and Dr Sahr are affiliated with the Department of Trauma Services, UnityPoint Health, Des Moines, Iowa. Mr Lamb, Ms Jackson, and Mr Oetting are affiliated with the Department of Respiratory Therapy, UnityPoint Health, Des Moines, Iowa.

Mr Lamb has disclosed relationships with Bayer and Medtronic. The other authors have disclosed no conflicts of interest. can lead to adverse hospital events, such as unplanned admission to the ICU, prolonged hospital stays, higher rates of mortality, and increased consumption of healthcare resources. ${ }^{1-15}$ Many adverse events are preventable. ${ }^{16,17}$
Dr Nyland presented a version of this report at the American Association for Respiratory Care Conference, held November 8, 2015, in Tampa, Florida.

Correspondence: Sarah K Spilman MA, Department of Trauma Services, UnityPoint Health, 1200 Pleasant Street, Des Moines, IA 50309. E-mail: sarah.spilman@unitypoint.org.

DOI: $10.4187 /$ respcare.04729 
There are numerous reasons why patients are at greater risk for pulmonary complications after trauma, especially patients who sustain blunt chest injury.9,14,18,19 Rib fractures, pulmonary contusions, and other chest conditions frequently impede respiratory function at several levels. First, fractures of the rib cage put the patient at a mechanical disadvantage, increasing the effort required with inspiration and decreasing maximal cough strength. . $^{8,14,19,20}$ Decreased lung volume and atelectasis may be the first events in a cascade leading to pulmonary complications. ${ }^{21}$ Second, the pain associated with these injuries makes patients unwilling or unable to take deep breaths unassisted. ${ }^{9,10,14,19,22}$ This can result in reduction in lung volumes for hours or even days. Third, trauma patients may experience extended periods of immobility or receive medications that depress respiratory drive and interfere with pulmonary function. ${ }^{12}$ Finally, preexisting health conditions, such as COPD or asthma, may be exacerbated after injury. ${ }^{8}$ It is notable that with an aging population, patients with preexisting pulmonary disease and age-related changes to the pulmonary system comprise a larger proportion of the trauma population, ${ }^{8}$ and attention must be given to how these preexisting conditions impact patient care.

\section{See the Related Editorial on Page 1708}

Protocols are an essential part of standardized medical care, and there is increased focus on evidence-based protocols for trauma patients. ${ }^{12}$ Lung expansion therapy and pulmonary hygiene are important aspects of respiratory care for hospitalized patients, with the ultimate aim of preventing respiratory complications rather than responding reactively after complications occur. ${ }^{12,23-26}$ However, support for prophylactic lung expansion therapy is mixed $^{8,10,23,25,27,28}$ and is often specific to certain patient populations, such as patients with cystic fibrosis ${ }^{29,30}$ or $\mathrm{COPD}^{31}$ and postoperative patients. ${ }^{21,32-34}$

There are 2 studies in the trauma literature that address prevention of pulmonary complications utilizing prophylactic respiratory therapy methods. In a sample of postoperative trauma and surgical subjects, Harbrecht et al ${ }^{12}$ found that implementation of a protocol for the early identification of patients at risk for pulmonary complications decreased ICU and hospital days. Hanlon et al ${ }^{35}$ also showed that initiation of a proactive pulmonary care regimen significantly reduced the number of unplanned upgrades in level of care and the number of intubations in a trauma population.

In addition to the limited number of studies pertaining to trauma patients, it is noted that the majority of research on proactive respiratory care has been conducted in ICU settings. What is neglected is work aimed at identifying patients on general in-patient wards who may be in danger

\section{QUICK LOOK}

\section{Current knowledge}

Trauma patients are at risk for respiratory complications after injury, and these complications can lead to longer hospital stays, unplanned admission to the ICU, and other adverse events. Prophylactic respiratory therapy has been found to prevent respiratory complications in some patient populations with known respiratory disease, but there is no consensus on whether this approach can reduce respiratory complications in a trauma population.

\section{What this paper contributes to our knowledge}

This study assessed the effectiveness of a proactive respiratory protocol for adult trauma subjects on an in-patient ward. Compared with a pre-protocol phase, the protocol was associated with reduced length of hospitalization, reductions in the use of bronchodilators, and fewer returns to the ICU for respiratory reasons. Protocol adherence was enhanced when respiratory therapists could direct the intervention.

of respiratory compromise, with the ultimate goal being prevention of respiratory complications and unnecessary admission to the ICU, especially for patients with thoracic and pulmonary injury or preexisting lung disease. The purpose of this study is to determine whether the adoption of prophylactic volume expansion therapy in the general in-patient setting can improve patient outcomes and reduce hospital stay in a population of trauma patients.

\section{Methods}

\section{Study Design and Patient Sample}

A study was conducted at a tertiary hospital with an adult Level 1 trauma center to determine the efficacy of a protocol to prevent ICU readmission for adult trauma patients on a specific ward in the hospital. The ward chosen for the study is the primary in-patient ward in the hospital for trauma, neurosurgical, and orthopedic patients. The study hospital is a 400-bed facility that serves a mediumsized metropolitan area as well as rural hospitals in a 100mile radius. The protocol was designed as a collaborative effort between trauma services and respiratory therapy (RT) to identify and treat patients at risk for pulmonary complications. Before the protocol, RT involvement in trauma care was not routinized and was initiated at the direction of the attending physician. The volume expansion protocol was initiated in October 2013. 


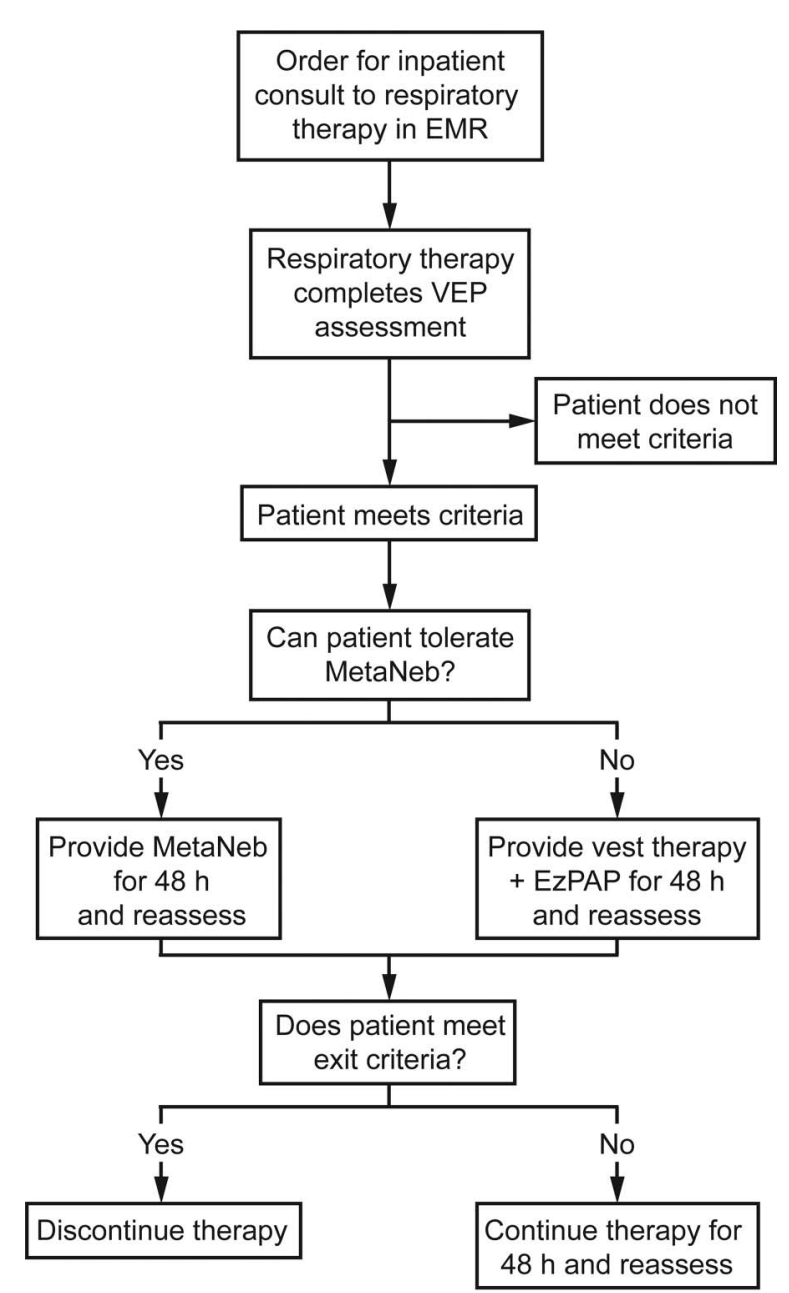

Fig. 1. Decision making algorithm for the volume expansion protocol (VEP). EMR = electronic medical record.

Protocol procedure required the trauma physician or resident to order an in-patient consultation to RT at the time the subject was admitted to the study ward (see Fig. 1); this included the transition from the ICU to the ward or admission from the emergency department to the ward. The respiratory therapist assessed the subject to determine whether volume expansion protocol inclusion criteria were met. Interim analyses were conducted in the fall of 2014 to compare outcomes from the first 6 months of the protocol (phase 1) with a 6-month period before protocol initiation (pre-protocol phase). It was determined that physician adherence to ordering the protocol was low and that protocol inclusion criteria were too broad. Efforts commenced to revise the protocol, improve the process for ordering the protocol, and reeducate physicians and staff. The revised protocol was introduced in February 2015 (phase 2).

To analyze the effectiveness of the current protocol, study inclusion criteria mirrored the revised protocol inclusion criteria from phase $2: \geq 3$ rib fractures; pulmonary contusions; exacerbation of COPD, asthma, or other doc- umented lung disease; or expected immobility of $\geq 48 \mathrm{~h}$ for subjects $\geq 65$ y old. Patients were excluded from this analysis if they had a tracheostomy at the time of admission to the ward. Three groups of subjects were compared: 50 subjects from the pre-protocol phase; the first 50 subjects who met study inclusion criteria in phase 1; and the first 50 subjects who met study inclusion criteria in phase 2 . Subjects were identified for the study if an assessment was documented in the medical record (phases 1 and 2); the trauma registry was used to identify subjects who would have met inclusion criteria in the pre-protocol phase.

All protocol assessments were completed by respiratory therapists at the bedside, and therapists determined the appropriate therapies using department guidelines and the protocol algorithm (see Fig. 1). Primary therapy was delivered through the MetaNeb 4.0 system (Hill-Rom Company, Batesville, Indiana), which combines lung expansion, secretion clearance, and aerosol delivery into a single therapy. The initial setting was $5 \mathrm{~cm} \mathrm{H}_{2} \mathrm{O}$, titrated to subject tolerance. If a subject could not tolerate MetaNeb, the subject was given vest therapy (The Vest System, model 205, Hill-Rom Company) with or without the EzPAP positive airway pressure system (Smiths Medical, St Paul, Minnesota). Other secondary therapies included Acapella vibratory positive expiratory pressure therapy system (Smiths Medical); Aerobika oscillating positive expiratory pressure therapy system (Monaghan Medical Corporation, Plattsburgh, New York); incentive spirometry; or tracheal and nasal suctioning. Therapy was conducted 4 times each day for $48 \mathrm{~h}$, and the subject was then reassessed. If the subject did not meet exit criteria, the therapy continued with reassessment at 48-h intervals. Exit criteria included the absence of active respiratory pathologies or risk factors for respiratory complications.

Chart reviews of the electronic medical record were conducted for data not included in RT databases or the trauma registry. The study was approved by the institutional review board at the hospital. The requirement of subject consent was waived because chart review occurred retrospectively after subject discharge.

\section{Study Variables}

Several demographic and diagnostic variables were extracted from the trauma registry, including sex, age, history of COPD, smoking history, injury mechanism of fall, payer source (commercial insurance, Medicare, Medicaid, other), whether the subject had an operative procedure during hospitalization, and hospital discharge disposition (home, skilled nursing facility). Body mass index was ascertained at the time of admission to the hospital. Injury diagnoses were extracted from the International Classification of Diseases, 9th Revision, Clinical Modification codes in the trauma registry and included chest injuries of 
Identifying Trauma Subjects at Risk for Respiratory Compromise

Table 1. Demographic and Diagnostic Characteristics of the Study Sample

\begin{tabular}{|c|c|c|c|c|}
\hline Characteristics & Pre-Protocol $(n=50)$ & Phase $1(n=50)$ & Phase $2(n=50)$ & $P$ \\
\hline Male sex, $n(\%)$ & $35(70)$ & $31(62)$ & $35(70)$ & .62 \\
\hline Age, median (IQR) y & $60(46-75)$ & $63(54-73)$ & $59(36-78)$ & .47 \\
\hline BMI, median (IQR) kg/m² & $28(24-33)$ & $29(25-34)$ & $28(23-33)$ & .77 \\
\hline History of COPD, $n(\%)$ & $7(14)$ & $9(18)$ & $6(12)$ & .59 \\
\hline Current smoker, $n(\%)$ & $8(16)$ & $16(32)$ & $14(28)$ & .16 \\
\hline Injury mechanism of fall, $n(\%)$ & $19(38)$ & $25(50)$ & $22(44)$ & .48 \\
\hline Injury severity score, $n(\%)$ & $19(10-25)$ & $13(9-17)$ & $10(9-19)$ & .002 \\
\hline \multicolumn{5}{|l|}{ Chest injury (not mutually exclusive), $n(\%)$} \\
\hline Rib fractures & $29(58)$ & $37(74)$ & $29(58)$ & .16 \\
\hline Pulmonary contusion & $22(44)$ & $12(24)$ & $9(18)$ & .01 \\
\hline Pneumothorax & $4(8)$ & $5(10)$ & $10(20)$ & .15 \\
\hline Flail chest & $2(4)$ & $2(4)$ & $0(0)$ & .36 \\
\hline \multicolumn{5}{|l|}{ Payer source (not mutually exclusive), $n(\%)$} \\
\hline Commercial insurance & $26(52)$ & $19(38)$ & $17(34)$ & .16 \\
\hline Medicare & $19(38)$ & $23(46)$ & $20(40)$ & .70 \\
\hline Medicaid & $1(2)$ & $4(8)$ & $10(20)$ & .01 \\
\hline Uninsured & $4(8)$ & $5(10)$ & $3(6)$ & .76 \\
\hline Operative procedure, $n(\%)$ & $14(28)$ & $7(14)$ & $7(14)$ & .12 \\
\hline Fracture fixation & $7(50)$ & $5(71)$ & $4(57)$ & .61 \\
\hline Spinal fusion & $4(29)$ & $0(0)$ & $3(43)$ & .14 \\
\hline Other & $3(21)$ & $2(29)$ & $0(0)$ & .24 \\
\hline Discharge to home, $n(\%)$ & $29(58)$ & $36(72)$ & $35(70)$ & .28 \\
\hline Discharge to skilled nursing facility, $n(\%)$ & $13(26)$ & $8(16)$ & $5(10)$ & .10 \\
\hline \multicolumn{5}{|l|}{$\begin{array}{l}N=150 . \\
\mathrm{IQR}=\text { interquartile range } \\
\mathrm{BMI}=\text { body mass index }\end{array}$} \\
\hline
\end{tabular}

rib fractures (807.03-807.19), flail chest (807.4), pneumothorax or pneumohemothorax $(860.0,860.1,860.4$, $860.5)$, and pulmonary contusion $(861.21,863.31)$. The injury severity score is based on the abbreviated injury score, with values ranging from 0 (no injury) to 75 (most severe).

Primary outcomes were hospital days, ward days, and ICU days. Unplanned admission to the ICU was ascertained from the medical record if a subject was transferred to the ICU after admission to the ward; we report the overall number as well as the number of subjects who were admitted to the ICU for respiratory reasons (eg, hypoxia or increased work of breathing). Additionally, we examined whether subjects were dispositioned directly from the emergency department to the ward, the use of bronchodilators (ipratropium bromide, albuterol) during hospitalization, and mortality.

\section{Statistical Procedures}

All analyses were performed with IBM SPSS Basic Statistics for Windows 20.0 (IBM Corp, Armonk, New York). Descriptive statistics were examined and are reported for continuous data as medians and interquartile ranges; cat- egorical data are reported as counts and percentages. Differences between medians were assessed using the KruskalWallis test by ranks, and nominal data were compared using Pearson chi-square test. Post hoc pairwise comparisons were used to determine where the differences existed between groups.

Ordinary least squares multiple regression analysis was used to determine whether the protocol was associated with decreased hospital stay. Histograms and analysis of residuals revealed that 2 subjects in the pre-protocol phase were outliers with hospital stays that exceeded $30 \mathrm{~d}$; these subjects were eliminated from the final regression model. The Durbin-Watson test was then used to test for autocorrelation. Initial regression models were tested with saturated main effects in the first step; the variables contributing least to the model were omitted, and only the final equation is presented. All statistical tests are 2-tailed and based on a .05 significance level.

\section{Results}

A total of 150 subjects were included in the study, with 50 subjects in each of the 3 phases. Table 1 shows the demographic and diagnostic characteristics of subjects in 


\section{IDENTIFying Trauma SubJects at Risk for Respiratory COMPROMise}

Table 2. Subject Outcomes

\begin{tabular}{|c|c|c|c|c|}
\hline Outcomes & Pre-Protocol $(n=50)$ & Phase $1(n=50)$ & Phase $2(n=50)$ & $P$ \\
\hline Hospital stay, median (IQR) d & $8(5-15)$ & $5(4-8)$ & $4(3-10)$ & .001 \\
\hline Ward stay, median (IQR) d & $6(4-10)$ & $4(3-7)$ & $4(2-7)$ & .001 \\
\hline ICU stay, median (IQR) d & $2(0-6)$ & $1(0-2)$ & $0(0-3)$ & .01 \\
\hline Discharged from emergency department to ward, $n(\%)$ & $16(32)$ & $28(56)$ & $25(50)$ & .043 \\
\hline Unplanned admission to ICU (all reasons), $n(\%)$ & $6(12)$ & $6(12)$ & $0(0)$ & .038 \\
\hline Unplanned admission to ICU (respiratory), $n(\%)$ & $4(8)$ & $4(8)$ & $0(0)$ & .12 \\
\hline Use of bronchodilators, $n(\%)$ & $16(32)$ & $8(16)$ & $9(18)$ & .11 \\
\hline Died, $n(\%)$ & $1(2)$ & $1(2)$ & $0(0)$ & .60 \\
\hline $\begin{array}{l}N=150 . \\
\mathrm{IQR}=\text { interquartile range }\end{array}$ & & & & \\
\hline
\end{tabular}

each phase of the study. Subjects in the pre-protocol phase had a significantly higher median injury severity score (19) than subjects in phase 1 (13) or phase 2 (10); post hoc pairwise comparisons indicate significant differences between the pre-protocol phase and phase $1(P=.02)$, as well as between the pre-protocol phase and phase 2 $(P=.003)$. More than one quarter of subjects in the preprotocol phase received an operative procedure during their hospital stay. This rate was reduced to $14 \%$ in both phase 1 and phase 2, but the difference across the phases was not statistically significant. Payer status was consistent across phases; however, there were significantly more subjects on Medicaid in phase 2 than in the other phases.

More than half of subjects in each phase had a diagnosis of rib fractures. Notably, a significantly higher percentage of subjects in the pre-protocol phase had a diagnosis of pulmonary contusion compared with the protocol phases. No other significant differences in diagnoses were noted between phases; nor were there any statistically significant differences across the phases in subject age, injury mechanism, comorbid medical conditions, or discharge disposition.

\section{Improvements in Subject Outcomes}

Table 2 shows that there was a significant decrease across the phases in hospital stay, with post hoc pairwise comparisons noting the main effects between pre-protocol and phase $1(P=.01)$ as well as between pre-protocol and phase $2(P=.001)$. There also was a significant decrease in ward days, with the most significant decrease between pre-protocol and phase $2(P=.001)$. ICU stay significantly decreased between pre-protocol and phase 1 $(P=.02)$.

More subjects were dispositioned from the emergency department to the ward in the protocol phases as compared with the pre-protocol phase. Additionally, the percentage of subjects with an unplanned admission to the ICU was reduced from $12 \%$ in the pre-protocol phase and phase 1 to
Table 3. Regression on Hospital Days

\begin{tabular}{lcr}
\hline \hline \multicolumn{1}{c}{ Characteristics } & $\beta$ & \multicolumn{1}{c}{$P$} \\
\hline Age & 0.04 & .036 \\
Injury severity score & 0.23 & $<.001$ \\
Unplanned admission to ICU & 6.25 & $<.001$ \\
Received operative procedure & 4.94 & $<.001$ \\
Respiratory protocol assessment & -1.62 & .053 \\
\hline
\end{tabular}

$0 \%$ in phase 2 . Bronchodilator use was lower in the protocol phases than in the pre-protocol phase, but the change was not statistically significant.

Because the injury severity score differed significantly between phases, multivariate regression was used to analyze the effect of the protocol on hospital stay. After 2 outliers were removed, analyses confirmed model fit $(P<.001)$. As shown in Table 3, receiving a protocol assessment significantly reduced duration of hospitalization, even after controlling for age, injury severity score, unplanned admission to the ICU, and operative procedures. It is noted that when all else is equal, subjects who met protocol inclusion criteria and received a volume expansion protocol assessment had a decrease in the overall stay by approximately $1.5 \mathrm{~d}$.

\section{Discussion}

A prophylactic respiratory protocol was associated with reduced hospital stay as well as an elimination of unplanned admission to the ICU in a population of trauma subjects. This is similar to prior findings in trauma populations ${ }^{12,35}$ and contradictory to claims that such protocols

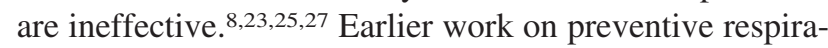
tory protocols has been conducted in patient populations with known respiratory disease (eg, COPD or cystic fibrosis), whereas this study examined a general trauma population at risk for respiratory compromise. Receiving a pro- 


\section{IDENTIFying Trauma SubJects at Risk for Respiratory COMPROMise}

tocol assessment decreased hospital stay by approximately $1.5 \mathrm{~d}$, even after controlling for important clinical factors.

In the pre-protocol phase and phase 1 , the rate of unplanned admission to the ICU was $12 \%$, which was higher than rates reported in the literature, which range from 4 to $5 \% .^{11,36}$ In phase 2 , none of the 50 subjects who met study inclusion criteria experienced an unplanned return to the ICU for any reason. This is a favorable finding, and further investigation is warranted to determine whether the improvement is sustainable. It is also notable that the protocol inclusion criteria in phase 2 were effective in identifying subjects who did not need prophylactic respiratory treatment. Only one subject in the phase 2 study period failed to meet protocol inclusion criteria and experienced an unplanned admission to the ICU for a respiratory cause. It was determined that the subject failed to meet protocol inclusion criteria due to incomplete documentation of the subject's respiratory disease history.

It is noteworthy that the median age of subjects in all phases of the study was approximately $60 \mathrm{y}$. Many trauma centers are experiencing increases in the geriatric population, and this population is also more vulnerable to pulmonary disease and respiratory complications. ${ }^{8,37}$ Chest trauma may exacerbate preexisting conditions and put this population at risk for adverse events. ${ }^{8}$ Identification and preventive treatment of at-risk patients is paramount when providing quality care to an aging population.

Because substantial improvements in outcomes were not noted after phase 1 , the study team revised the protocol and collected an additional phase of data. We believe the insignificant findings from phase 1 were due to a variety of factors. First, when protocol initiation was at the discretion of the physician, only half of eligible subjects received an order for the protocol. Subjects with rib fractures were more likely to have the protocol ordered than those without this diagnosis, which suggests that providers may have ordered the protocol for subjects with thoracic injuries and not ordered the protocol for those perceived ineligible. Results from phase 2 demonstrated that protocol adherence was improved when it was initiated by the physician or the respiratory therapist. This is consistent with results from other studies that found adherence may be higher when respiratory therapists play a role in initiating protocols. $^{38,39}$

Second, the number of protocol inclusion criteria was reduced in phase 2 . It was determined that phase 1 criteria were overly inclusive and captured subjects without increased risk for respiratory compromise. For example, all patients with an ICU stay of $\geq 48 \mathrm{~h}$ before admission to the ward were included in phase 1 , regardless of chest injury or expected immobility. The limited criteria from phase 2 not only achieved the desired results but also used valuable hospital resources more efficiently.
Two additional findings were noteworthy and unexpected. First, more subjects were dispositioned from the emergency department to the ward as compared with the pre-protocol phase. This may be the result of lower patient acuity but also may reflect increased physician comfort to admit patients directly to the ward because of the availability of respiratory therapists to promptly evaluate and monitor patients. Second, study results show an important reduction in the use of bronchodilators, including albuterol and ipratropium. Before protocol initiation, there were no standards for RT involvement in trauma patient care, and there was inconsistency in the manner in which patients received respiratory treatments. The use of nebulized bronchodilators may have been used as a proxy to bring RT to the bedside after patients experienced worsening respiratory processes, such as shortness of breath or increased work of breathing; this is consistent with literature that documents the overprescription of bronchodilators by physicians. ${ }^{38}$ With the respiratory protocol, subjects were evaluated by RT before they experienced respiratory problems, and appropriate treatments were ordered for the subjects' specific conditions. Bronchodilators are not without side effects, and routine administration of bronchodilators is not recommended, ${ }^{25,38,39}$ so this reduction is a favorable finding.

The study has several limitations. First, some data were collected retrospectively, and we could only ascertain information that was documented in the electronic medical record. Second, low physician adherence to ordering the protocol in phase 1 was partially a limitation of the electronic medical record. If a patient was receiving RT treatments in the ICU, the protocol was not issued automatically at the time of transfer to the ward. Efforts are under way at the study hospital to improve the process for ordering the protocol so that all trauma patients are evaluated by a respiratory therapist at the time of admission to the ward.

Third, the pre-protocol population had a higher median injury severity score than and more operative procedures than subjects in either study phase (see Table 1). Although the majority of demographic and diagnostic characteristics were similar across the study phases, subjects in the preprotocol phase were considered more injured than subjects in the protocol phases. However, multivariable regression controlled for injury severity score and other clinical factors, and results still suggest that receiving the protocol assessment resulted in decreased hospital stay.

Finally, we echo the conclusion of Harbrecht et al ${ }^{12}$ that it is difficult to know whether the reduced stay was directly due to the respiratory protocol or other factors. As Andrews et $\mathrm{al}^{23}$ have noted, clinical care is complex, and many factors can impact clinical outcomes; this makes it difficult to ascertain the effect of specific interventions. Additionally, results are derived from a single trauma cen- 


\section{IDENTIFying Trauma SubJects at Risk for Respiratory COMPROMise}

ter, and further investigation is warranted to determine whether similar results are achievable in a different setting. We believe our findings are supportive of the respiratory protocol, but replication is required to determine whether clinical improvements were due to the protocol or simply due to chance.

\section{Conclusion}

This study demonstrates that a prophylactic respiratory therapy protocol improved outcomes for trauma subjects at a Level 1 trauma center. Identifying patients at risk for respiratory compromise can mitigate adverse events and ultimately improve the course of the hospital stay.

\section{ACKNOWLEDGMENTS}

We thank K Danielle Hahn (UnityPoint Health) for assistance with data collection.

\section{REFERENCES}

1. Afshar M, Smith GS, Cooper RS, Murthi S, Netzer G. Trauma indices for prediction of acute respiratory distress syndrome. J Surg Res 2016;201(2):394-401.

2. Antonelli M, Moro ML, Capelli O, De Blasi RA, D’Errico RR, Conti $\mathrm{G}$, et al. Risk factors for early onset pneumonia in trauma patients. Chest 1994;105(1):224-228.

3. Battle CE, Hutchings H, Evans PA. Risk factors that predict mortality in patients with blunt chest wall trauma: a systematic review and meta-analysis. Injury 2012;43(1):8-17.

4. Battle C, Hutchings H, Lovett S, Bouamra O, Jones S, Sen A, et al. Predicting outcomes after blunt chest wall trauma: development and external validation of a new prognostic model. Crit Care 2014;18(3): R98.

5. Becher RD, Colonna AL, Enniss TM, Weaver AA, Crane DK, Martin RS, et al. An innovative approach to predict the development of adult respiratory distress syndrome in patients with blunt trauma. J Trauma Acute Care Surg 2012;73(5):1229-1235.

6. Hoyt DB, Simons RK, Winchell RJ, Cushman J, HollingsworthFridlund P, Holbrook T, Fortlage D. A risk analysis of pulmonary complications following major trauma. J Trauma 1993;35(4):524531.

7. Navarrete-Navarro P, Rodriguez A, Reynolds N, West R, Habashi N, Rivera R, et al. Acute respiratory distress syndrome among trauma patients: trends in ICU mortality, risk factors, complications and resource utilization. Intensive Care Med 2001;27(7):1133-1140.

8. Ramly E, Kaafarani HM, Velmahos GC. The effect of aging on pulmonary function: implications for monitoring and support of the surgical and trauma patient. Surg Clin North Am 2015;95(1):53-69.

9. Simon B, Ebert J, Bokhari F, Capella J, Emhoff T, Hayward T 3rd, et al. Management of pulmonary contusion and flail chest: an Eastern Association for the Surgery of Trauma practice management guideline. J Trauma Acute Care Surg 2012;73(5):S351-S361.

10. Unsworth A, Curtis K, Asha SE. Treatments for blunt chest trauma and their impact on patient outcomes and health service delivery. Scand J Trauma Resusc Emerg Med 2015;23:17.

11. Fakhry SM, Leon S, Derderian C, Al-Harakeh H, Ferguson PL. Intensive care unit bounceback in trauma patients: an analysis of unplanned returns to the intensive care unit. J Trauma Acute Care Surg 2013;74(6):1528-1533
12. Harbrecht BG, Delgado E, Tuttle RP, Cohen-Melamed MH, Saul MI, Valenta CA. Improved outcomes with routine respiratory therapist evaluation of non-intensive-care-unit surgery patients. Respir Care 2009;54(7):861-867.

13. Makris N, Dulhunty JM, Paratz JD, Bandeshe H, Gowardman JR Unplanned early readmission to the intensive care unit: a case-control study of patient, intensive care and ward-related factors. Anaesth Intensive Care 2010;38(4):723-731.

14. Pharaon KS, Marasco S. Mayberry J. Rib fractures, flail chest, and pulmonary contusion. Curr Trauma Rep 2015;1(4):237-242.

15. Stelfox HT, Bagshaw SM, Gao S. Characteristics and outcomes for hospitalized patients with recurrent clinical deterioration and repeat medical emergency team activation. Crit Care Med 2014;42(7):1601-1609.

16. Marquet K, Claes N, De Troy E, Kox G, Droogmans M, Schrooten W, et al. One fourth of unplanned transfers to a higher level of care are associated with a highly preventable adverse event: a patient record review in six Belgian hospitals. Crit Care Med 2015;43(5):1053-1061.

17. Vlayen A, Verelst S, Bekkering GE, Schrooten W, Hellings J, Claes N. Incidence and preventability of adverse events requiring intensive care admission: a systematic review. J Eval Clin Pract 2012;18(2):485-497.

18. Miller PR, Croce MA, Kilgo PD, Scott J, Fabian TC. Acute respiratory distress syndrome in blunt trauma: identification of independent risk factors. Am Surg 2002;68(1):845-850; discussion 850-851.

19. Vana PG, Neubauer DC, Luchette FA. Contemporary management of flail chest. Am Surg 2014;80(6):527-535.

20. Easter A. Management of patients with multiple rib fractures. Am J Crit Care 2001;10(5):320-327.

21. Lawrence VA, Cornell JE, Smetana GW. Strategies to reduce postoperative pulmonary complications after noncardiothoracic surgery: systematic review for the American College of Physicians. Ann Intern Med 2006;144(8):596-608.

22. Dhillon TS, Galante JM, Salcedo ES, Utter GH. Characteristics of chest wall injuries that predict postrecovery pulmonary symptoms: a secondary analysis of data from a randomized trial. J Trauma Acute Care Surg 2015;79(2):179-186; discussion 186-187.

23. Andrews J, Sathe NA, Krishnaswami S, McPheeters ML. Nonpharmacologic airway clearance techniques in hospitalized patients: a systematic review. Respir Care 2013;58(12):2160-2186.

24. Fink JB. Forced expiratory technique, directed cough, and autogenic drainage. Respir Care 2007;52(9):1210-1221; discussion 1221-1223.

25. Strickland SL, Rubin BK, Drescher GS, Haas CF, O'Malley CA, Volsko TA, et al. AARC clinical practice guideline: effectiveness of nonpharmacologic airway clearance therapies in hospitalized patients. Respir Care 2013;58(12):2187-2193.

26. Volsko TA. Airway clearance therapy: finding the evidence. Respir Care 2013;58(10):1669-1678.

27. Pasquina $\mathrm{P}$, Tramèr MR, Granier JM, Walder B. Respiratory physiotherapy to prevent pulmonary complications after abdominal surgery. Chest 2006;130(6):1887-1899.

28. Restrepo RD, Braverman J. Current challenges in the recognition, prevention and treatment of perioperative pulmonary atelectasis. Expert Rev Respir Med 2015;9(1):97-107.

29. Flume PA, Robinson KA, O'Sullivan BP, Finder JD, Vender RL, Willey-Courand DB, et al. Cystic fibrosis pulmonary guidelines: airway clearance therapies. Respir Care 2009;54(4):522-537.

30. Lester MK, Flume PA. Airway-clearance therapy guidelines and implementation. Respir Care 2009;54(6):733-750; discussion 751-753.

31. Vargas F, Bui HN, Boyer A, Salmi LR, Gbikpi-Benissan G, Guenard $\mathrm{H}$, et al. Intrapulmonary percussive ventilation in acute exacerbations of COPD patients with mild respiratory acidosis: a randomized control trial. Crit Care 2005;9(4):R382-R389.

32. Chumillas S, Ponce JL, Delgado F, Viciano V, Mateu M. Prevention of postoperative pulmonary complications through respiratory reha- 


\section{Identifying Trauma Subjects at Risk for Respiratory Compromise}

bilitation: a controlled clinical study. Arch Phys Med Rehabil 1998; 79(1):5-9.

33. Liao G, Chen R, He J. Prophylactic use of noninvasive positive pressure ventilation in post-thoracic surgery patients: a prospective randomized control study. J Thorac Dis 2010;2(4):205-209.

34. Pelosi P, Jaber S. Noninvasive respiratory support in the perioperative period. Curr Opin Anaesthesiol 2010;23(2):233-238.

35. Hanlon VD, White F, Hustosky AE, McCormick CA, Hayes EJ, Wilson A. Benefits of standardizing additional airway clearance in the trauma patient population. J Trauma Nurs 2014;21(3):127-132.

36. Ang DN, Rivara FP, Nathens A, Jurkovich GJ, Maier RV, Wang J, MacKenzie EJ. Complication rates among trauma centers. J Am Coll Surg 2009;209(5):595-602.
37. Adams SD, Cotton BA, McGuire MF, Dipasupil E, Podbielski JM, Zaharia A, et al. Unique pattern of complications in elderly trauma patients at a Level 1 trauma center. J Trauma Acute Care Surg 2012;72(1):112-118

38. Kallam A, Meyerink K, Modrykamien AM. Physician-ordered aerosol therapy versus respiratory therapist-driven aerosol protocol: the effect on resource utilization. Respir Care 2013;58(3): 431-437.

39. Kollef MH, Shapiro SD, Clinkscale D, Cracchiolo L, Clayton D, Wilner R, Hossin L. The effect of respiratory therapist-initiated treatment protocols on patient outcomes and resource utilization. Chest 2000;117(2):467-475.

This article is approved for Continuing Respiratory Care Education credit. For information and to obtain your CRCE

(free to AARC members) visit 